\title{
Wir werden immer älter
}

\author{
Heinz Rüddel \\ Psychosomatische Fachklinik, St. Franziska-Stift, Bad Kreuznach, Deutschland
}

Ein ganzes Paket von Faktoren scheint dafür verantwortlich zu sein, dass wir immer älter werden. Präventive und arbeitsmedizinische Maßnahmen wie der Nichtraucherschutz und die Verbesserung der Arbeitsbedingungen sind ebenso bedeutsam wie die Sekundärprävention kardiovaskulärer Erkrankungen und die Primärprävention von wichtigen Tumorerkrankungen wie Brustkrebs, Hodenkrebs und Darmkrebs. Auch die verbesserte medizinische Diagnostik und bessere Behandlungsmöglichkeiten sind relevant.

Innerhalb der Prävention unterscheiden wir seit einigen Jahrzehnten allgemeine Primärpräventionsmaßnahmen wie z.B. die Verbesserung des Nichtraucherschutzes oder die gezielte Prävention bei Risikogruppierungen und die Sekundärprävention, um den Verlauf von bereits eingetretenen Erkrankungen günstig zu beeinflussen, z.B. nach einem Herzinfarkt. Dabei ist das Ziel natürlich nicht allein eine quantitative Lebensverlängerung, sondern dass die gewonnenen Lebensjahre von möglichst hoher Lebensqualität sind.

In der Prävention aktive Berufsgruppen sind unter anderem Ärzte und Psychologen. Ärzte in Praxen und Institutionen, Betriebsärzte und Arbeitsmediziner sowie Gesellschaften und Akademien etc. sind in den letzten 2 Jahrzehnten auch in der Präventionsmedizin deutlich aktiver geworden, als sie es in den 1970er und 1980er Jahren noch waren. Auch Psychologen und insbesondere psychologische Psychotherapeuten greifen zunehmend präventive Aspekte auf. Sowohl bei Psychologen als auch bei Ärzten steigt die Forschungsaktivität auf dem Gebiet der präventiven Maßnahmen und zwar sowohl in der Rehabilitationsforschung als auch in der Gesundheitspsychologie, der Klinischen Psychologie und Psychotherapie, der Biologischen Psychologie sowie der Kinder- und Jugendlichenpsychotherapie [Schuster und Rüddel, 2010].

Auch wenn gesellschaftlicher Konsens darüber besteht, dass der Prävention immer stärkere Bedeutung zukommt, sind wichtige Rahmenbedingungen unklar. Ein Präventionsgesetz wird seit vielen Jahren diskutiert, ist aber noch nicht verabschiedet, und die finanziellen Regelungen sind suboptimal.

\section{Ziele von präventiven Maßnahmen}

Präventive Maßnahmen sollen folgende Ziele haben:

- Hinführung von Kindern und Jugendlichen zu einem gesundheitsbewussten Lebensstil.

- Erhaltung bzw. Verbesserung der beruflichen Leistungsfähigkeit im mittleren und höheren Alter.

- Zugewinn an gesunden Lebensjahren mit hoher Lebensqualität im Alter [Scholl und Albrecht, 2010].

Die solideste Datenlage zum Effekt und zur Bedeutsamkeit präventiver Maßnahmen liegt für den Bereich der koronaren Herzerkrankung und der arteriosklerotischen Veränderungen vor. Herzerkrankungen sind die führende Todesursache in den USA und in den meisten Ländern der Welt. Im Jahr 2006 waren in den USA $68 \%$ der Todesfälle auf koronare Herzerkrankungen zurückzuführen. Unverändert spielen bei der Prävention dabei die Risikofaktoren erster Ordnung, also hohe Serumlipide, arterielle Hypertonie, Nikotinkonsum und Diabetes (bzw. mit Diabetes assoziierte Probleme wie Übergewicht und Adipositas) eine entscheidende Rolle, da alle diese vier Risikofaktoren effektiv veränderbar sind und Änderungen des Lebensstils dabei eine wesentliche Bedeutung zukommt. Seit dem Jahr 2000 ist es in den USA gelungen, die herzkreislaufbasierten Todesraten bei Männern um 22\% und bei Frauen um 23\% zu reduzieren. Genauere Analysen zur Modifikation von Risikofaktoren betonen aber in den letzten Jahren sehr deutlich die Bedeutung von Gender (Geschlecht), Kultur und sozialer Schicht. So ist es bei Armen generell schwieriger, effektiv präventiv $\mathrm{zu}$ arbeiten [Braun und O'Connor, 2010]. Insbesondere scheint es fast unmöglich $\mathrm{zu}$ sein, bei Personen unterhalb der Armutsgrenze LDL-Cholesterin zu senken. Aber auch der Zugang zu einer guten Behandlung der koronaren Herzerkrankung ist offensichtlich von der sozialen Schicht abhängig [Yeh et al., 2010].

Was soll mit diesem Themenheft erreicht werden?

Im vorliegenden Themenheft werden einige neuere Entwicklungen in der Prävention aufgegriffen. Es wird sehr deutlich,

\section{KARGER}

Fax +497614520714

Information@Karger.de

www.karger.com
(C) 2010 S. Karger GmbH, Freiburg

Accessible online at:

www.karger.com/ver
Prof. Dr. Heinz Rüdde

Psychosomatische Fachklinik, St. Franziska Stift

Postfach 1553, 55505 Bad Kreuznach, Deutschland

Tel. +49 671 8820-2001, Fax- 190

h.rueddel@fskh.de 
wie schwierig und teilweise dürftig die empirische Datenlage in vielen Präventionsbereichen ist. In einem Interview mit Christian Dormann, Professor für Arbeits-, Organisationsund Wirtschaftspsychologie an der Universität Mainz, werden Probleme und Chancen der Prävention in einer sich verändernden Arbeitswelt aufgezeigt. Nadine Schuster legt anhand einer sehr großen Untersuchung mit 2300 Managern im Finanz- und Bankwesen dar, wie wichtig sowohl die erlebte organisationale Unterstützung als auch die empfundene Wertschätzung und Anerkennung vonseiten des Betriebes für die Prävention von Burnout-Syndromen ist.

In einem anderen Schwerpunkt dieses Themenheftes werden Möglichkeiten der Prävention im Kindes- und Jugendalter dargestellt. Von der Arbeitsgruppe um Charlotte Hanisch und Manfred Döpfner wird dargestellt, wie zusätzlich zu den statistischen Methoden einer Wirksamkeitsprüfung von Präventions- und Interventionsprogrammen deren klinische Bedeutsamkeit aufgezeigt werden kann. Dabei wird klinische Bedeutsamkeit derart definiert, dass ein vorher auffälliges pathologisches Merkmal nach der Intervention nicht mehr im pathologischen Bereich liegt, sondern im Bereich der Normalpopulation.
Aus der Arbeitsgruppe um Brunna Tuschen-Caffier wird eine Übersichtsarbeit vorgelegt, die deutlich macht, dass eine konsequente und frühzeitig einsetzende indizierte Prävention der sozialen Angststörung (soziale Phobie) im Kindes- und Jugendalter der Entwicklung von Angststörungen im Erwachsenenalter vorbeugen könnte. Nina Heinrichs, Tanja Zimmermann und Peter Herschbach untersuchen, ob Kinder aus Familien, in denen die Mütter an einer malignen gynäkologischen Erkrankung leiden, Verhaltensauffälligkeiten entwickeln. Die bisherige Datenlage lässt jedoch noch keine Schlussfolgerungen zu für die Notwendigkeit von Präventionsmaßnahmen in dieser Zielgruppe.

Die Leser mögen sich in ihrem jeweiligen Arbeitsgebiet, insbesondere wenn sie als psychologische und ärztliche Psychotherapeuten tätig sind, selbstkritisch fragen, wie weit sie in der Behandlungsplanung bei ihren Patientinnen oder Patienten neben der durchgeführten Psychotherapie auch präventive Aspekte adäquat berücksichtigen. Aber auch Kliniker und Wissenschaftler sollen sich etwas stärker bewusst werden, wie schwierig und teilweise dürftig die Datenlage in der klinischen oder experimentellen Präventionsforschung ist. Hier besteht noch erheblicher Forschungsbedarf.

\section{Literatur}

Brown JR, O'Connor GT: Coronary heart disease and prevention in the United States. N Engl J Med 2010;362:2150-2153.

Scholl J, Albrecht C: Wie wollen wir alt werden? Dtsch Ärztebl 2010;107:B1436-1437.
Schuster N, Rüddel H: Prävention und Rehabilitation; in Hiller W, Leibing E, Leichsenring F, Sulz SKD (Hrsg): Lehrbuch der Psychotherapie, Bd 1: Wissenschaftliche Grundlagen der Psychotherapie. München, CIP, 2010, pp 235-248.
Yeh RW, Sidney S, Chandra M, Sorel M, Selby JV, Go AS: Population trends in the incidence and outcomes of acute myocardial infarction. N Engl J Med 2010;362:2155-65. 